\section{Corticoides para el tratamiento de COVID-19}

\author{
MAURICIO SALINAS ${ }^{1}$
}

\section{Corticosteroids treatment for COVID-19}

'Departamento de Medicina Oriente, Facultad de Medicina. Universidad de Chile. Santiago, Chile.

Correspondencia a: Mauricio Salinas Jose M Infante 717. Providencia. Santiago, Chile. mrsalinas@uchile.cl
L: a historia del uso de corticoides en las enfermedades respiratorias es un largo recuento de éxitos y fracasos. Así como la efectividad de éstos en asma está demostrada y es indudable, en diversas otras condiciones el uso de corticoides, inicialmente esperanza de mejoría, ha terminado por demostrar efectos deletéreos e incluso mayor mortalidad, como ocurrió con el uso en pacientes con fibrosis pulmonar idiopática ${ }^{1}$.

Probablemente es en el ámbito de las infecciones y el síndrome de distrés respiratorio del adulto (SDRA) donde la información es más controvertida. El uso de corticoides en infecciones virales respiratorias ha sido probado en múltiples ocasiones sin resultados positivos, incluyendo los estudios en enfermedades previas similares al COVID-19, MERS y SARS ${ }^{2}$, en Hantavirus ${ }^{3}$ y en influenza donde, el uso de éstos se ha asociado con mayor mortalidad en los casos graves y críticos ${ }^{4}$. En SDRA luego de múltiples estudios negativos, un trabajo multicéntrico publicado a principios de año, demostró beneficio del uso de dexametasona en dosis altas en una población muy seleccionada de enfermos con este síndrome ${ }^{5}$. Este estudio demoró casi seis años en obtener la muestra y fue detenido por lento reclutamiento, por lo cual parece prudente esperar que los resultados se repliquen antes de considerarlo un estándar.

En el tratamiento de pacientes con COVID19 los corticoides aparecen hoy como la opción terapéutica, luego de demostrarse la falta de eficacia de hidroxicloroquina, lopinavir/ritonavir, tocilizumab y con resultados muy cuestionables para el plasma convaleciente, lo que incluso ha llevado al comité de expertos del instituto nacional de salud (NIH) de los estados unidos a emitir una recomendación en contra de su uso ${ }^{6}$.

En el primer número de septiembre de la revista JAMA se acaban de publicar tres ensayos clínicos de terapia esteroidal en pacientes graves con COVID-1 $9^{7-9}$ y un meta análisis ${ }^{10}$, que incluye los mencionados ensayos. El meta análisis muestra que los corticoides tienen un beneficio en mortalidad a 28 días en pacientes críticos con COVID-19.

Pese a que el beneficio de los corticoides en pacientes graves y en ventilación mecánica parece claro con el agregado de información disponible, existen observaciones relevantes en los datos publicados. Los resultados del meta-análisis están influidos fuertemente por los datos del estudio RECOVERY ${ }^{11}$, que es 59\% de los sujetos de análisis. Los tres ensayos incluidos en el mismo número decidieron detener su reclutamiento luego de la publicación del estudio RECOVERY, con los reportados problemas descritos cuando esto ocurre ${ }^{12}$. Así, dos de esos estudios, realizados con hidrocortisona, no lograron demostrar beneficio. Otro estudio agregado a posterior en el análisis suplementario, con metilprednisolona, tampoco demostró beneficios ${ }^{13}$. Quizás uno de los elementos más criticables, es que el estudio RECOVERY no registró eventos adversos, lo cual deja un gran incógnita en ese aspecto.

La historia de los corticoides en COVID-19 no terminará acá. Muchas preguntas permanecen abiertas, quizás una de las más relevantes es si el efecto beneficioso es exclusivo de dexametasona o aplica a todos los corticoides. Los datos publicados hasta ahora solo apoyan el uso de dexametasona. Otras preguntas importantes son: ¿cuál es la duración óptima del tratamiento esteroidal?, ¿los pacientes no críticos, se benefician de esta terapia?, ¿pacientes con bajos requerimientos de oxígeno?, ¿pacientes sin necesidad de oxígeno?, ¿el paciente que no responde o se deteriora en segunda instancia debe recibir más corticoides?.

Este es un espacio aún abierto para desarrollar estudios que ayuden a precisar la utilidad de estos fármacos en cuanto a dosis y tiempos. El uso de cor- 
ticoides está asociado a múltiples efectos adversos, sobre todo cuando la terapia es prolongada o en dosis elevadas. Algunos de los factores de riesgo para adquirir COVID-19 como obesidad, edad mayor y diabetes mellitus, conllevan mayor posibilidad de eventos adversos con el uso de corticoides. En los pacientes que sobreviven a un SDRA se ha reportado la miopatía como secuela relevante y limitante en seguimientos de largo plazo ${ }^{14}$, lo cual también puede ser agravado por el tratamiento esteroidal.

En el enfrentamiento de la pandemia actual, el mayor desafío sanitario para la humanidad desde la segunda guerra mundial, los profesionales de la salud han debido enfrentar escenarios de extrema adversidad, carencia de recursos, falta de información y ausencia de terapias científicamente sustentadas. La pléyade de tratamientos utilizados sin mayor sustento en los últimos meses, favorecidos por información que circula en redes sociales o la prensa y que después culminan con estudios negativos, ha demostrado que solo el desarrollo de investigación de buena calidad permitirá tomar decisiones correctas. El llamado es a no guiar las decisiones terapéuticas por datos anecdóticos y prejuicios, errores ya cometidos con el uso de hidroxicloroquina, que solo van en desmedro de la buena práctica médica y del desarrollo de medicina basada en evidencia ${ }^{15}$.

\section{Referencias}

1. Raghu G, Anstrom KJ, King TE, Lasky JA, Martinez FJ. Prednisone, Azathioprine, and N-Acetylcysteine for Pulmonary Fibrosis. N Engl J Med 2012; 366 (21): 1968-77.

2. Russell CD, Millar JE, Baillie JK. Clinical evidence does not support corticosteroid treatment for 2019-nCoV lung injury. Vol. 395, The Lancet. Lancet Publishing Group; 2020. p. 473-5.

3. Vial PA, Valdivieso F, Ferres M, Riquelme R, Rioseco ML, Calvo M, et al. High-dose intravenous methylprednisolone for hantavirus cardiopulmonary syndrome in Chile: A double-blind, randomized controlled clinical trial. Clin Infect Dis 2013; 57 (7): 943-51.

4. Ni YN, Chen G, Sun J, Liang BM, Liang ZA. The effect of corticosteroids on mortality of patients with influenza pneumonia: A systematic review and meta-analysis. Crit Care 2019; 23 (1).

5. Villar J, Ferrando C, Martínez D, Ambrós A, Muñoz T, Soler JA, et al. Dexamethasone treatment for the acute respiratory distress syndrome: a multicentre, randomised controlled trial. Lancet Respir Med 2020; 8 (3): 267-76.
6. Panel C-19 TG. Statement on Convalescent Plasma EUA | Coronavirus Disease COVID-19 [Internet]. September 01, 2020. [cited 2020 Sep 13]. Available from: https://www.covid19treatmentguidelines.nih.gov/ statement-on-convalescent-plasma-eua/

7. Tomazini BM, Maia IS, Cavalcanti AB, Berwanger O, Rosa RG, Veiga VC, et al. Effect of Dexamethasone on Days Alive and Ventilator-Free in Patients With Moderate or Severe Acute Respiratory Distress Syndrome and COVID-19: The CoDEX Randomized Clinical Trial. JAMA. Published online September 2, 2020. doi: 10.1001/jama.2020.17021.

8. Writing Committee for the REMAP-CAP Investigators. Effect of Hydrocortisone on Mortality and Organ Support in Patients With Severe COVID-19: The REMAP-CAP COVID-19 Corticosteroid Domain Randomized Clinical Trial. JAMA. Published online September 2, 2020. doi: 10.1001/jama.2020.17022.

9. Dequin P-F, Heming N, Meziani F, Plantefève G, Voiriot G, Badié J, et al. Effect of Hydrocortisone on 21-Day Mortality or Respiratory Support Among Critically Ill Patients With COVID-19: A Randomized Clinical Trial. JAMA. Published online September 2, 2020. doi: 10.1001/jama.2020.16761.

10. WHO Rapid Evidence Appraisal for COVID-19 Therapies (REACT) Working Group, Sterne JAC, Murthy S, Diaz J V, Slutsky AS, Villar J, et al. Association Between Administration of Systemic Corticosteroids and Mortality Among Critically Ill Patients With COVID-19: A Meta-analysis. JAMA. Published online September 2, 2020. doi: 10.1001/jama.2020.17023.

11. Dexamethasone in Hospitalized Patients with Covid-19. Preliminary Report. N Engl J Med. Published online July 17, 2020.doi: 10.1056/NEJMoa2021436.

12. Guyatt GH, Briel M, Glasziou P, Bassler D, Montori VM. Problems of stopping trials early. BMJ 2012; 344 (7865).

13. Jeronimo CMP, Farias MEL, Val FFA, Sampaio VS, Alexandre MAA, Melo GC, et al. Methylprednisolone as Adjunctive Therapy for Patients Hospitalized With COVID-19 (Metcovid): A Randomised, Double-Blind, Phase IIb, Placebo-Controlled Trial. Clin Infect Dis 2020; ciaa1177. doi:10.1093/cid/ciaa1177.

14. DInglas VD, Aronson Friedman L, Colantuoni E, Mendez-Tellez PA, Shanholtz CB, Ciesla ND, et al. Muscle Weakness and 5-Year Survival in Acute Respiratory Distress Syndrome Survivors. Crit Care Med 2017; 45 (3): 446-53.

15. Serpa Neto A, Hodgson C. Will Evidence-based Medicine Survive the COVID-19 Pandemic?. Vol. 17, Annals of the American Thoracic Society. NLM (Medline); 2020. p. 1060-1. 